\title{
Molecular basis for amyloid fibril formation and stability
}

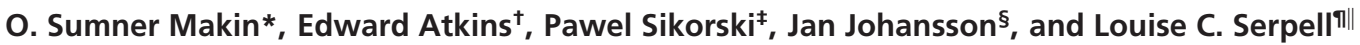

*Structural Medicine, Department of Haematology, University of Cambridge, Cambridge Institute for Medical Research, Hills Road, Cambridge CB2 2XY, United Kingdom; 'Department of Polymer Science and Engineering, University of Massachusetts, Amherst, MA 01003; 'Department of Physics, Norwegian University of Science and Technology, NO-7491 Trondheim, Norway; §Department of Molecular Biosciences, Swedish University of Agricultural Science Biomedical Center, Box 575, S-751 23 Uppsala, Sweden; and "Department of Biochemistry, John Maynard-Smith Building, School of Life Sciences, University of Sussex, Falmer BN1 9QG, United Kingdom

Edited by Alexander Rich, Massachusetts Institute of Technology, Cambridge, MA, and approved November 30, 2004 (received for review September 15, 2004)

The molecular structure of the amyloid fibril has remained elusive because of the difficulty of growing well diffracting crystals. By using a sequence-designed polypeptide, we have produced crystals of an amyloid fiber. These crystals diffract to high resolution ( 1 Å) by electron and x-ray diffraction, enabling us to determine a detailed structure for amyloid. The structure reveals that the polypeptides form fibrous crystals composed of antiparallel $\boldsymbol{\beta}$-sheets in a cross- $\boldsymbol{\beta}$ arrangement, characteristic of all amyloid fibers, and allows us to determine the side-chain packing within an amyloid fiber. The antiparallel $\boldsymbol{\beta}$-sheets are zipped together by means of $\pi$-bonding between adjacent phenylalanine rings and salt-bridges between charge pairs (glutamic acid-lysine), thus controlling and stabilizing the structure. These interactions are likely to be important in the formation and stability of other amyloid fibrils.

$\mathrm{x}$-ray diffraction $\mid$ side-chain packing $\mid$ structure $\mid \pi-\pi$ bonding $\mid \beta$-sheet interaction

$\mathbf{T}$ he importance of amyloid fibrils in disease has meant that, in recent years, extended $\beta$-sheet structure has become prominent in the literature. Amyloid fibrils are found in many different fatal diseases, including Alzheimer's and the spongiform encephalopathies (e.g., Creutzfeldt-Jakob disease). Each disease is characterized by a particular protein or polypeptide that aggregates in an ordered manner to form insoluble amyloid fibrils. These amyloid fibrils are deposited in the tissues, where they are associated with the pathology of the disease. It is well established that amyloid is predominantly composed of a $\beta$-sheet structure (1) based on the detailed structure deduced for cross- $\beta$ silk (2). The cross- $\beta$ structure and texture is a robust, stable structure in which the protein chains are held together securely by repetitive hydrogen-bonding that extends the length of the fibrils.

Amyloid fibrils can be self-assembled in vitro from many different polypeptides and proteins, many of which are involved in disease. It has been postulated that the generic amyloid conformation, the cross- $\beta$ structure (2), may be a universal, energetic minimum for aggregated proteins $(3,4)$. Once formed, amyloid fibrils are extremely stable and difficult to solubilize (5).

Structural studies of amyloid fibrils have focused primarily on those composed of short polypeptides. These fibrils may be assembled in vitro yielding well ordered fibers that are substantially more amenable to study with x-ray diffraction, electron microscopy, and solid-state NMR. Structural studies have suggested that these synthetic amyloid fibrils are composed of a core $\beta$-sheet structure in which the $\beta$-strands run orthogonal to the fiber axis and that the frequent and repetitive hydrogen-bonding extends parallel to the fiber axis (6). The fibers are thought to be composed of two or more $\beta$-sheets held together by hydrophobic surface contacts or specific electrostatic interactions. Nanocrystals of a polypeptide have yielded some additional information concerning the arrangement of the $\beta$-strands within an amyloid-forming polypeptide (7). However, the exact conformation remains unknown.

We have used a designed 12-mer peptide to investigate the underlying structure of amyloid fibrils. The polypeptide sequence is KFFEAAAKKFFE (referred to hereafter as AAAK) and contains a 4-mer motif at either end with a hydrophobic region in the center designed to inhibit the formation of a $\beta$-turn (8). Motifs within the original peptide are found in the sequences of notable amyloidogenic peptides. In particular, phenylalanine pairs occur in the Alzheimer's disease peptide amyloid- $\beta$ and in the amyloidosisrelated serum amyloid A (Table 1). A motif of three consecutive alanine residues and the motif AAXK are found throughout the repeat region of the Parkinson's disease-related protein, $\alpha$-synuclein (Table 1). Proteins that form amyloid fibrils do not show any obvious sequence similarities, yet all form similar structures. However, many amyloid-forming peptides have aromatic residues thought to play an important role in fibrillogenesis (9).

We have established that the AAAK polypeptide forms well ordered amyloid-like fibrils that assemble laterally in the intersheet direction to form three-dimensional crystals (Fig. 1a). This added dimensional order has allowed us to collect high-resolution x-ray (Fig. 2a) and electron diffraction data (Fig. $2 b$ ) from the fibrous crystals. Thus, these high-resolution diffraction data have enabled the determination of a fibril structure, including the side-chain arrangements, allowing the detailed nature of the intersheet interactions within the amyloid fibril to be visualized. We believe that the structure presented here can be taken to be representative of the generic structure for amyloid.

\section{Methods}

Fiber/Crystal Formation and Electron Microscopy. The peptide AAAK was synthesized as described in ref. 10, and fibrillogenesis was achieved by shaking a $3 \mathrm{mM}$ concentration of peptide in PBS at $37^{\circ} \mathrm{C}$ for 1 week. The solution (diluted $1 / 10$ ) was examined by using transmission electron microscopy. A droplet ( $4 \mu \mathrm{l})$ was placed on carbon-coated, glow-discharged copper grids (400 mesh; TAAB, Aldermaston, U.K.) and blotted. The grid was washed twice with $4 \mu \mathrm{l}$ of filtered $(0.2 \mu \mathrm{m})$ water and then stained by using $2 \%$ (wt/vol) uranyl acetate. The grids were examined with a Philips 208 electron microscope operated at $80 \mathrm{kV}$.

Electron Diffraction. The grid that had been prepared for transmission electron microscopy was examined in a Philips EM400T electron microscope by electron diffraction. A second identically prepared grid was platinum-coated and used as a calibrant.

This paper was submitted directly (Track II) to the PNAS office. Abbreviation: AAAK, KFFEAAAKKFFE.

Data deposition: The atomic coordinates have been deposited in the Protein Data Bank www.pdb.org (PDB ID code 2BFI).

|To whom correspondence should be addressed. E-mail: I.c.serpell@sussex.ac.uk.

C 2005 by The National Academy of Sciences of the USA 
Table 1. Amyloid protein sequences highlighting similarities to the designed peptide AAAK

\begin{tabular}{lll} 
Sequence of peptide & \multicolumn{1}{c}{ Parent protein } & \multicolumn{1}{c}{ Pathology } \\
\hline KLVFFAE & Amyloid- $\beta$ protein & Alzheimer's disease \\
SFFSFLGEAFD & Serum amyloid protein & Chronic inflammation amyloidosis \\
NFGAIL & Islet amyloid protein & Type II diabetes \\
PQGGYQQYN & PrP & Creutzfeldt-Jakob disease \\
SFNNGDCCFILD & Gelsolin & Finnish inflammation amyloidosis \\
NFGSVQFV & Lactadherin & Aortic medial amyloid \\
FAIRHF and FENKFAV & ABri & British dementia \\
AAAXK & $\alpha$-Synuclein & Parkinson's disease \\
DWSFYLYYTEFT & $\beta-2$ microglobulin & Dialysis related amyloidosis \\
DFNKF & Calcitonin & Medullary carcinoma of thyroid \\
\hline
\end{tabular}

$\mathrm{X}$ designates variable residues or no residues. Bold characters designate aromatic residues.

Electron diffraction was carried out by using the microscope operated at $100 \mathrm{kV}$ and camera lengths of 295-2,300 mm. The electron diffraction patterns were recorded on Kodak film and a real-space image was taken of the selected area. The films were scanned with an Epson digitizer.

Diffraction patterns were examined and processed with the CLEARER image processing suite (O.S.M. and L.C.S., unpublished results). The diffractograms were processed to remove the background and increase the contrast to bring up weaker diffraction peaks (Fig. $2 c$ ). The image was divided into single-pixelwide annuli, and a contrast stretch was performed on each annulus based on the maximum and minimum pixel values in the selected circular region.

X-Ray Diffraction. Fibers were partially aligned by using two methods. First, a fiber sample was prepared by using a stretch frame as described in ref. 11. Secondly, a thin-film or mat was prepared by using a cryoloop. A cryoloop $0.5 \mathrm{~mm}$ in diameter normally used for freezing crystals was dipped into the solution of aggregated AAAK polypeptide forming a thin film across the loop, which was allowed to dry, resulting in a thin layer of fibers at many orientations within the plane of the film.

Data collection was carried out in house by using a Rigaku rotating anode, $\mathrm{CuK} \alpha \mathrm{x}$-ray source, and MARresearch image plate or by using synchrotron radiation at European Synchrotron Radiation Facility (Grenoble, France) (Beamline BL4/ID2; wavelength $=0.99 \AA$ ). Several images at various specimen-tofilm distances were collected.

X-ray diffraction data were examined by using MOSFLM (A. Leslie, Laboratory of Molecular Biology, University of Cam-

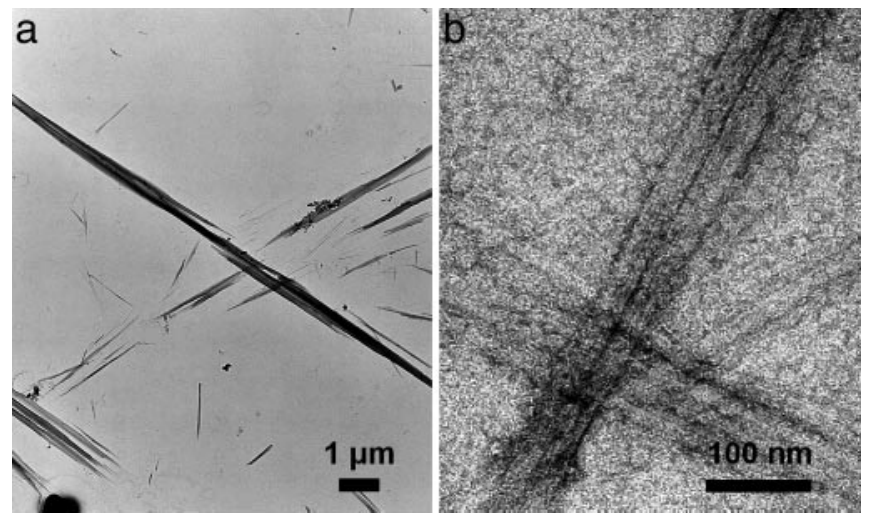

Fig. 1. Electron micrographs of fibrous crystals. The AAAK polypeptide assembles after incubation in PBS. Negative-stained transmission electron microscopy shows fibrous crystals in a field at low magnification (a) and the elongated crystals at higher magnification showing the $50-\AA$ striations running parallel to the fiber axis $(b)$. bridge) run on a LINUX workstation, and the positions of the diffraction signals were noted (Table 2). The diffraction data showed the classic cross- $\beta$ spacings at $4.76 \AA$ (meridian) and 10.6 $\AA$ (equator). Many other reflections were also observed (Table 2). The lowest resolution signal was observed at $24.4 \AA$ on the equator. No reflection was observed at $48 \AA$, despite data collection extending to $<100 \AA$. The diffractogram was further processed by using the CLEARER image processing suite. The circularly symmetric background was removed in the following manner. The image was centered and divided up into singlepixel-wide annuli; for each annulus, the position of the 10th percentile boundary was determined, and its value was subtracted from every pixel in that annulus. The positions of peaks were determined by using a one-dimensional image calculated by circularly averaging regions of the image with the same (rounded) radius within a user-supplied range of angles. Local averaging of the one-dimensional image and differentiating to find maxima established the approximate peak locations. The precise peak positions were found by fitting a parabola to the region surrounding the approximate peak locations in the nonlocally averaged, one-dimensional image.

Interpretation of Diffraction Data and Modeling. X-ray (Table 2) and electron diffraction signals were indexed to a space group and unit cell. The results were confirmed by applying a grid search algorithm by using starting values derived from known constraints from the polypeptide. Single polypeptide chains were built by using INSIGHT II (Accelrys, San Diego) run on an SGI workstation, in an ideal $\beta$-sheet configuration and in such a manner as to prevent overlaps. Initial models were refined manually by using CERIUS 2 (Accelrys). Details of the structure were modeled carefully to prevent the clashing of side chains. Clash scores calculated by using MOLPROBITY (12), and visual inspection allowed the comparison of different rotamer permutations. Side-chain positions were refined by energy minimization in CERIUS 2 and, finally, with molecular dynamics by using DISCOVER (Accelrys). Diffraction patterns were simulated from the models by using CERIUS 2 and CLEARER and compared to observed diffraction patterns.

\section{Results}

Examination of the Fibrillar Assemblies by Electron Microscopy. A solution of the AAAK polypeptides was incubated over several days. Examination by electron microscopy revealed fibrous crystals. The diameter of the fibrous crystals varied from $\approx 120$ to $2,400 \AA$, with lengths in the micrometer range (Fig. 1a). The fibers have narrowly spaced striations running parallel to the axis of the fibers, suggestive of smaller protofilaments (Fig. 1b). The diameter of these protofilaments was measured to be $\approx 50 \AA$, consistent with measurements from other amyloid fibrils (13). 

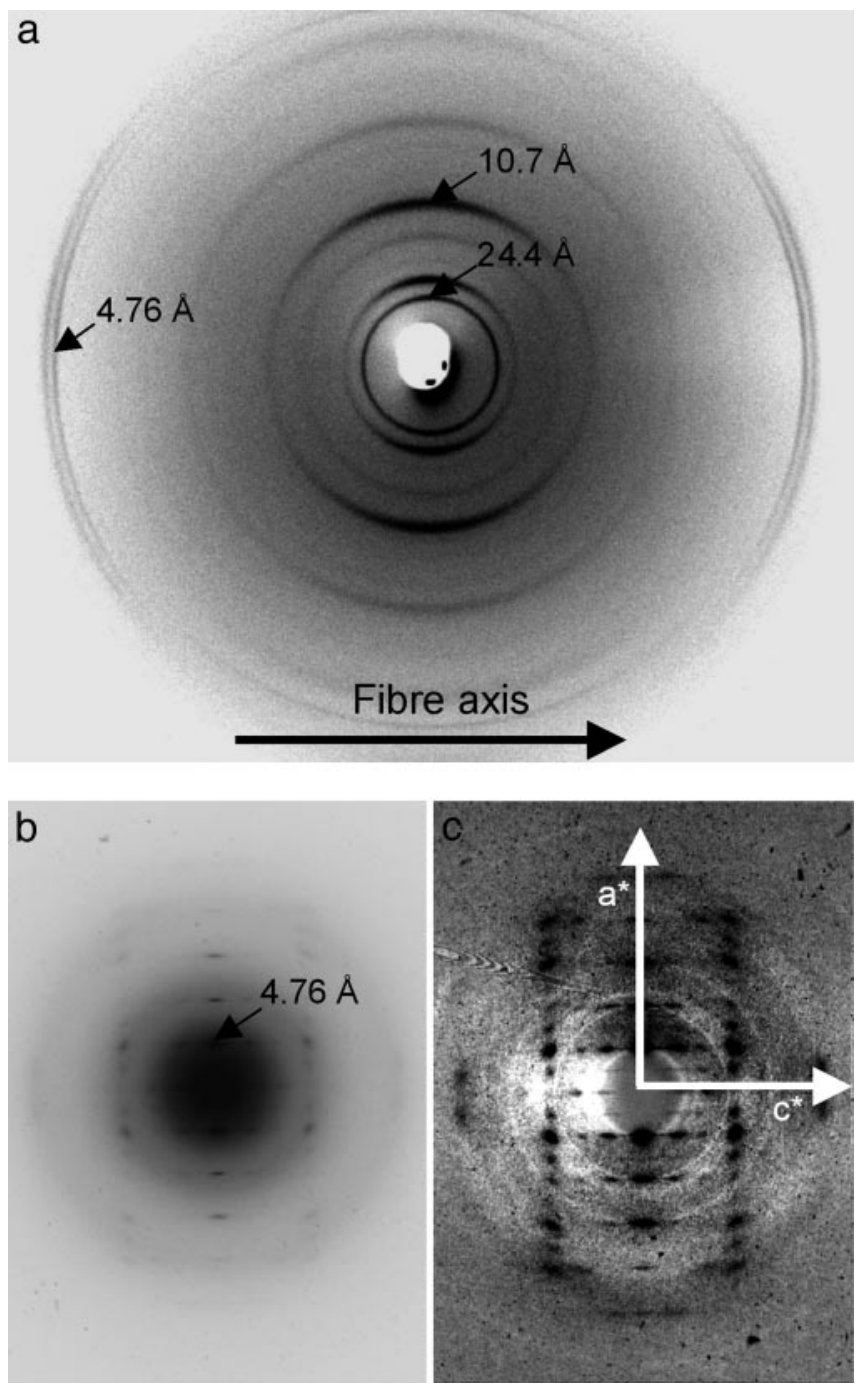

Fig. 2. Diffraction data from fibrous crystals. (a) X-ray diffraction pattern obtained by using synchrotron radiation from partially aligned, bundled fibers. The fiber axis is horizontal with the incident beam directed orthogonal to the fiber axis. Diffraction spacings were observed to a resolution beyond 2 $\AA$ and are shown in Table 2. ( $b$ and $c$ ) Electron diffraction patterns obtained from the fibrillar crystals with the incident beam parallel to the [ $\left[\begin{array}{lll}0 & 1 & 0\end{array}\right]$ zone axis reveal high-resolution, crystalline order before $(b)$ and after $(c)$ contrast enhancement. Discrete diffraction signals were observed out to a resolution of $0.9 \AA$.

X-Ray and Electron Diffraction from Fibrous Nanocrystals. Highresolution x-ray diffraction (beyond $2 \AA$ ) (Fig. $2 a$ ) and electron diffraction patterns (beyond $0.9 \AA$ ) (Fig. $2 b$ and $c$ ) enabled the structure of the polypeptide arranged in an amyloid fibril to be solved. The diffraction patterns showed the characteristic cross- $\beta$ reflections (Fig. $2 a$ and Table 2) consistent with amyloid fibrils (6). The equatorial diffraction signals (Fig. 2a) are sharper than is commonly observed for amyloid fibrils, probably arising from the added intersheet order within the large crystallites. The lowresolution data from the x-ray diffraction pattern (Fig. $2 a$ ) contribute essential information about the overall arrangement of the peptides in the crystal structure and add to information concerning the intersheet direction not present in the electron diffraction data.

Indexing and Interpretation of Diffraction Data. The sharp diffraction signals from the x-ray diffraction patterns (Fig. $2 a$ ) allowed the unit cell to be deduced and the signals to be indexed (Table 2 and
Table 2. Comparison of observed diffraction signals with signals calculated from the structure

\begin{tabular}{|c|c|c|c|c|}
\hline \multirow{2}{*}{$\begin{array}{l}\text { Measured } \\
\text { resolution, } \AA\end{array}$} & \multicolumn{3}{|c|}{ Miller indices } & \multirow{2}{*}{$\begin{array}{l}\text { Predicted } \\
\text { resolution, A }\end{array}$} \\
\hline & $h$ & $k$ & $I$ & \\
\hline 24.43 & 0 & 0 & 2 & 24.06 \\
\hline 19.94 & 0 & 1 & 1 & 19.49 \\
\hline 13.27 & 0 & 1 & 3 & 12.82 \\
\hline 10.66 & 0 & 2 & 0 & 10.66 \\
\hline 9.33 & 1 & 0 & 1 & 9.34 \\
\hline 8.69 & 1 & 1 & 0 & 8.70 \\
\hline 7.93 & 0 & 2 & 4 & 7.98 \\
\hline 7.19 & 0 & 2 & 5 & 7.14 \\
\hline 6.13 & 1 & 0 & 6 & 6.14 \\
\hline 5.65 & 1 & 3 & 1 & 5.66 \\
\hline 5.33 & 0 & 4 & 0 & 5.33 \\
\hline 4.91 & 1 & 3 & 5 & 4.90 \\
\hline 4.76 & 2 & 0 & 0 & 4.76 \\
\hline 4.66 & 1 & 0 & 1 & 4.65 \\
\hline 4.37 & 1 & 3 & 7 & 4.38 \\
\hline 4.00 & 0 & 0 & 12 & 4.01 \\
\hline 3.91 & 1 & 1 & 11 & 3.91 \\
\hline 3.79 & 1 & 5 & 3 & 3.78 \\
\hline 3.44 & 0 & 0 & 14 & 3.44 \\
\hline 2.95 & 2 & 5 & 6 & 2.95 \\
\hline 2.71 & 3 & 1 & 9 & 2.71 \\
\hline 2.61 & 1 & 7 & 8 & 2.61 \\
\hline 2.46 & 3 & 5 & 5 & 2.46 \\
\hline 2.44 & 2 & 7 & 6 & 2.44 \\
\hline 2.36 & 3 & 6 & 1 & 2.36 \\
\hline 2.30 & 1 & 9 & 1 & 2.30 \\
\hline 2.26 & 3 & 0 & 15 & 2.26 \\
\hline 2.20 & 3 & 5 & 11 & 2.20 \\
\hline 2.18 & 1 & 9 & 7 & 2.18 \\
\hline 2.09 & 1 & 6 & 18 & 2.08 \\
\hline
\end{tabular}

Fig. 2a). The diffraction data could be indexed by using $\mathrm{P} 2{ }_{1} 2_{1} 2_{1}$ space group and unit cell with dimensions $a=9.52 \AA, b=21.3 \AA$, and $c=48.1 \AA$. The dimensions are defined as $a$, the hydrogen bonding direction; $b$, the $\beta$-sheet ribbon-stacking direction; and $c$, the peptide chain direction. These values match the expected unit cell for a cross- $\beta$ structure in an antiparallel conformation (see Fig. 4) (2). Electron diffraction patterns demonstrate that the cell is orthorhombic, because the $a$ and $c$ axes are clearly orthogonal (Fig. $2 c$ ). This detailed pattern is taken down the [0 010$]$ zone axis, indicating that the direction of $b$ is fixed and orthogonal to $a$ and $c$. Unit-cell dimensions were established by indexing the x-ray diffraction data by using information from the electron diffractograms and the dimensions of a single peptide chain.

Strong meridional signals are observed at $4.76 \AA$ in both $\mathrm{x}$-ray and electron diffraction patterns (Fig. 2). These signals arise from the spacing between hydrogen-bonded $\beta$-strands within a $\beta$-sheet. A layer line at $9.5 \AA$ is observed in the electron diffraction data and indicates that the polypeptide chains adopt an antiparallel $\beta$-sheet arrangement and gives a cell dimension of $a=9.5 \AA$. A signal is also observed in the x-ray diffraction pattern at $9.3 \AA$ and likely arises from the layer line. There is no signal observed at $9.5 \AA$ (Table 2), the expected position of the meridional reflection $\left[\begin{array}{lll}1 & 0 & 0\end{array}\right]$.

X-ray diffraction data show a strong equatorial signal at 10.6 $\AA$ that arises from the intersheet spacing. The $10.7 \AA$ signal and other signals (Table 2) can be indexed to a $b$ cell length of 21.3 $\AA$. Electron diffraction data shows very strong, even layer-lines, indicating that there are at least two chains in the unit cell, which requires the $b$ unit cell dimension of $2 \times 10.7 \AA$.

A 12 -mer peptide in an extended $\beta$-conformation would 


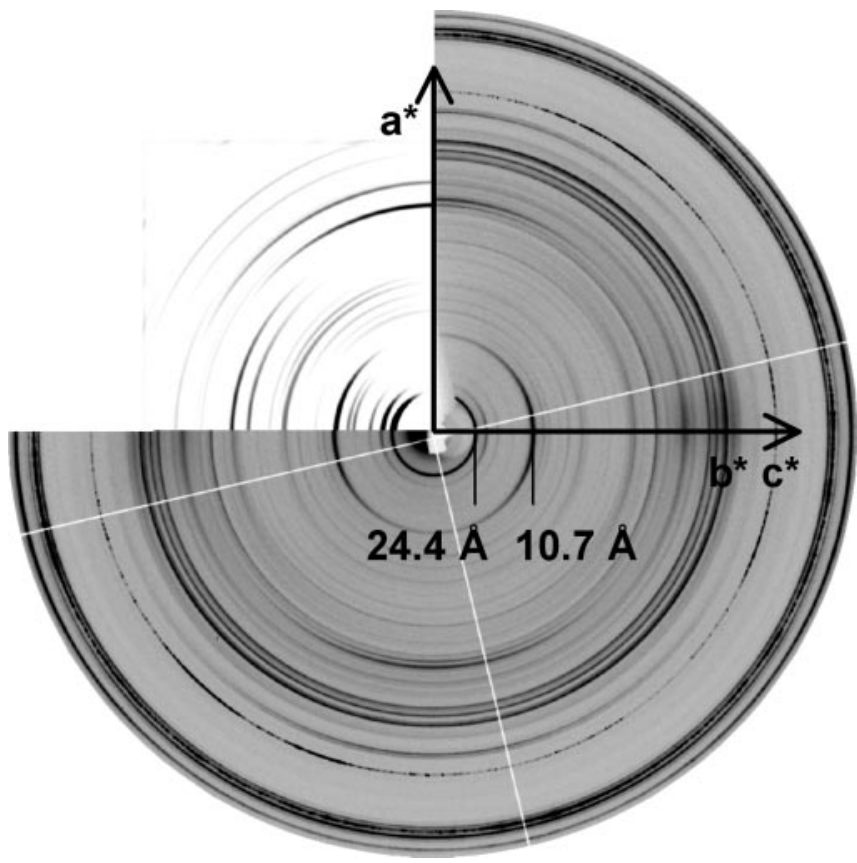

Fig. 3. Comparison of observed and calculated x-ray diffraction patterns The observed $x$-ray diffraction pattern from the partially aligned fibrous crystals is shown with the top left quadrant showing the calculated diffraction pattern. The calculated pattern is simulated from the model structure. It is clear where the observed and calculated diffraction peaks match, and this shows excellent agreement between diffraction signal positions.

measure $\approx 3.5 \times 12=42 \AA$. A strong diffraction signal is observed on the equator in the x-ray diffraction pattern at $24 \AA$. Fitting of the $x$-ray diffraction signals indicated that they are consistent with a cell length, $c$, of $48 \AA$, and modeling shows that this is very suitable to contain the polypeptide chains.

Assignment of the space group took place during the modeling phase. Nevertheless, several absences were noted. Neither x-ray nor electron diffraction data show a signal on the meridian at 9.5 $\AA$ [ $\left.\begin{array}{lll}1 & 0 & 0\end{array}\right]$. Likewise, no x-ray signal at $21.3 \AA\left[\begin{array}{lll}0 & 1 & 0\end{array}\right]$ is observed on the equator. Low-angle diffraction data (data not shown) shows that the $\left[\begin{array}{lll}0 & 0 & 1\end{array}\right]$ signal at $48 \AA$ is not present. This observation is consistent with $\mathrm{P} 2{ }_{1} 2_{1} 2_{1}$, which has systematic absences at odd $h 00,0 k 0$ and $00 l$. Table 2 and Fig. 3 demonstrate the strong correlation between the observed signals and those calculated on the basis of the aforementioned space group and unit cell.

Structural Determination of the AAAK Amyloid Fiber. The unit cell dimensions imply that the cell contains four chains. A model was built by first considering a single chain, then a pair of antiparallel strands, and, finally, two pairs of strands. After constructing a single chain, a pair of antiparallel strands was built. The absence of a signal at $\left[\begin{array}{lll}1 & 0 & 0\end{array}\right]$ and weakness of the odd layer-lines arises from the $2_{1}$ symmetry of this arrangement (13). The aromatic side chains are closely interlocked because they are substantially wider than the 4.7- $\AA$ spacing between hydrogen-bonded $\beta$-strands. Each phenylalanine side chain has four possible rotamer conformations. Hence, for two adjacent antiparallel strands, there are 256 permutations within each block of interlocked aromatics at each end of the chain. This number is reduced because of symmetry constraints. Considering periodicity along the chain $(a)$ direction but only a single pair of antiparallel $\beta$-strands, the high density of aromatic groups meant that only one permutation was reasonable. Hence, a pair of antiparallel chains was modeled with probable phenylalanine rotamers established, thereby showing intermeshed aromatic rings.
We then considered the whole unit cell comprised of two pairs of antiparallel chains separated by the $\beta$-sheet distance of $10.6 \AA$, as required by the $b$ cell dimension. The relative shift of the second pair of chains in the $a c$ plane and its axis of rotation were explored. Automated construction of these models allowed rapid analysis of different arrangements of peptide chains within the unit cell. Many hundreds of models were generated in this manner. Each model was then inspected carefully for clashing, and, for each, an x-ray and electron diffraction pattern was simulated and compared with those observed. The absence of a 48 - $\AA$ signal in the low-angle x-ray data greatly constrained the possible shifts along the $c$ direction. The final model gave the highest similarity between the calculated and observed diffraction data of all of the models examined. This structure had $\mathrm{P} 22_{1} 2_{1} 2_{1}$ symmetry, and, after manual adjustments, side-chain positions were refined by energy minimization as described in Methods.

The Architecture of the Amyloid Core. The final model structure was elucidated by precise modeling and by comparison of observed and calculated diffraction data (Fig. 3). The polypeptide chains are arranged within the crystal by applying the space-group symmetry to a single chain, which results in a face-centered arrangement of chains in the $b c$ plane (Fig. 4d). In the crystal structure (Fig. 4), the extended polypeptide $\beta$-strands run orthogonal to the fibril axis and are hydrogen-bonded in an antiparallel arrangement in the fiber direction, forming long $\beta$-sheet ribbons (Fig. $4 b$ ). The $\beta$-sheet ribbons are parallel and in register and are arranged in a staggered manner shifted in the chain direction (c) by $c / 2$ (Fig. $4 c$ and $d$ ). This structure creates an overlapping, brick-like appearance when viewed down the fibril axis $(a)$ (Fig. $4 d$ and $f$ ). A fibrillar crystal structure can be generated from the repeating unit of a single extended $\beta$-strand (Fig. 4e). This arrangement resembles previously suggested structural models for amyloid fibrils $(1,13-15)$. In this structure, we can clearly see how the $\beta$-sheets are held together by the interactions between side chains (Fig. $4 c$ and $d$ ). The $\mathrm{N}$ terminal half of one $\beta$-strand molecule associates with the $\mathrm{C}$ terminal half of another $\beta$-strand molecule, allowing the aromatic side chains to closely interlock but preventing the overlapping of aliphatic side chains. It is clear from the structure (Fig. $4 d$ and $f$ ) that this arrangement may provide space for the binding of sodium (0.95- $\AA$ ionic radius) or chloride ions (1.67- $\AA$ ionic radius). Charged pairings of the lysine and glutamic acid residues extend alternately along the $\beta$-sheets. This pairing is likely to be responsible for the antiparallel arrangement of the $\beta$-strands. Phenylalanine interactions between adjacent stands are dominated by parallel-displaced rings; whereas, between sheets, the aromatic rings form a T shape (16) (Fig. $4 c$ and $d$ ). The parallel-displaced arrangement of aromatic side chains has been shown to be the preferred packing in proteins that is highly stable (17). The staggered arrangement of the polypeptide chains creates a horizontal banding pattern when the structure is viewed along the $b$ axis (Fig. $4 b$ and $e$ ). This structure may provide an explanation of the striations observed in electron micrograph images (Fig. 1b).

The tightly packed arrangement of side chains between the $\beta$-sheets is likely to be responsible for the crystalline nature of the material and the high quality of the observed diffraction data. The result is a very stable and rigid structure. It is likely that the AAAK central motif in the polypeptide stabilizes an extended conformation and may be responsible for the efficient formation of amyloid fibril crystals from this peptide, because self-association of the extended peptide would be energetically favorable. The AAAK peptide has a net charge of +1 at neutral $\mathrm{pH}$. A net-charge of \pm 1 has been shown to be essential for fibril formation from designed hexapeptides (18).

\section{Discussion}

A striking feature of the structure presented here is the use of phenylalanine side chains to stabilize the intersheet packing by 

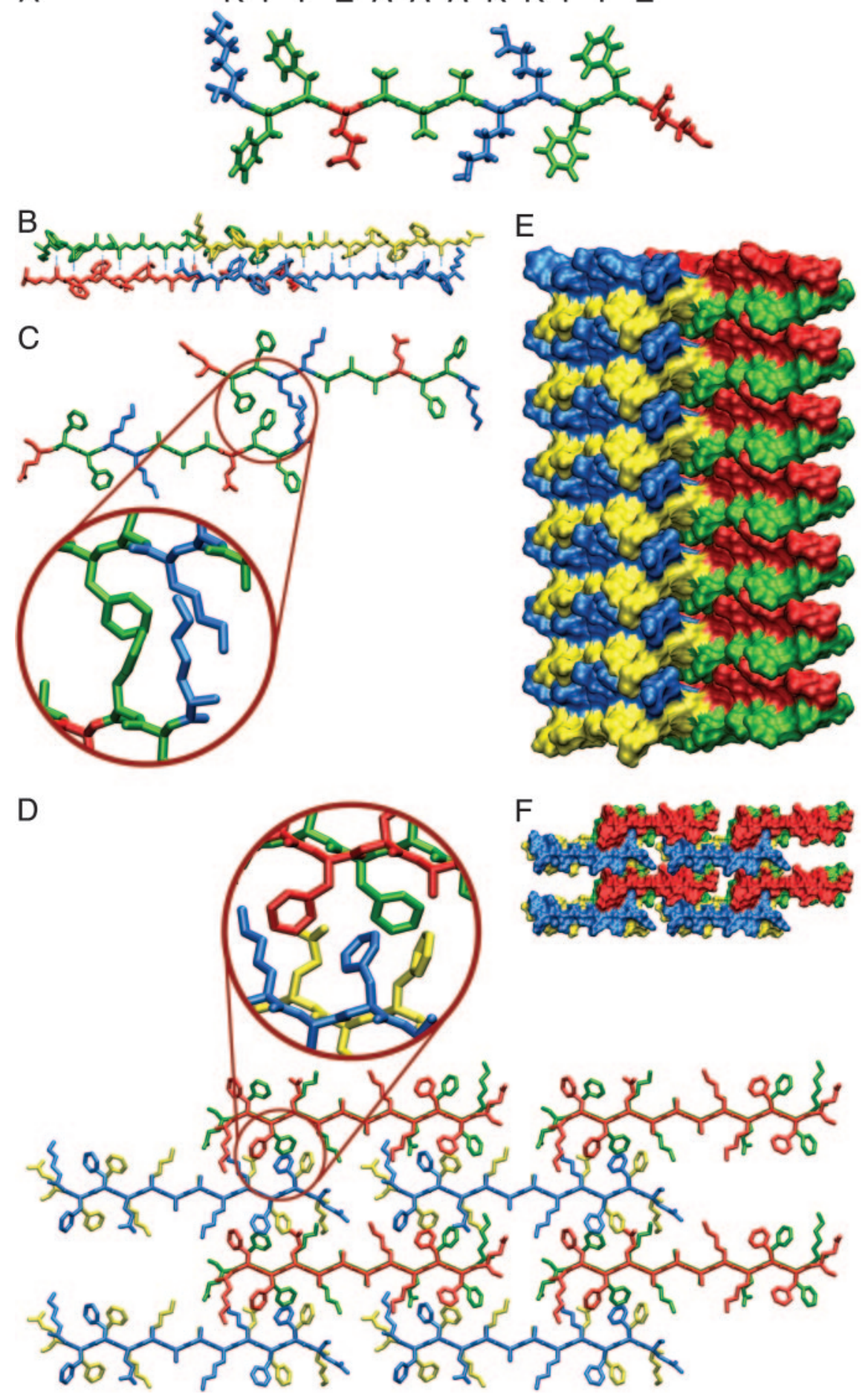

Fig. 4. The structure of the 12-mer peptide colored by residue type (basic, blue; acidic, red; nonpolar, green) or by chain. The structure is generated from a single peptide by imposing the $\mathrm{P} 22_{1} 2_{1} 2_{1}$ symmetry, which creates a brick-like arrangement of overlapping peptide molecules (shown in $d$ ) and results in a very rigid structure held together with hydrogen-bonding, $\pi-\pi$-bonding, and electrostatic interactions. (a) A single 12-mer peptide labeled with residue names. (b) Four chains (colored blue, green, yellow, and red) showing hydrogen-bonding between the pairs of chains. (c) Two chains colored by residue type, with a higher-magnification image highlighting the intersheet $\pi-\pi$ aromatic interactions. (d) Crystal packing with differently colored chains. The higher-magnification image highlights a cluster of phenylalanine side chains showing intersheet interactions and stacking between hydrogen-bonded $\beta$-strands. (e) Surface representation of crystal packing elongated along the fiber axis to show the close packing of the structure. ( $f$ ) A view down the a axis colored by chain.

means of $\pi-\pi$ stacking (Fig. $4 c$ and $d$ ). The importance of aromatic rings in amyloidogenesis has been demonstrated in many peptides (9) (Table 1). Previous studies examining the importance of different side chains in tetrapeptides revealed that the tetrapeptide
KFFE was able to form amyloid-like fibrils (10). These experiments also highlighted the importance of charge attraction, because peptides KFFK and EFFE could not form fibrils alone but only when mixed in equimolar quantities (10). The central hydrophobic 
cluster of amyloid- $\beta$ (residues 17-21 LVFFA) has been particularly implicated in amyloid fibril formation (19). Tjernberg et al. (20) found that the fragment QKLVFF binds to amyloid $\beta$ to prevent amyloidogenesis, whereas follow-up studies investigated the short peptides, LVFFA (21) and LPFFD (22), and showed that they are also inhibitors and that the fragment KLVFFAE forms well ordered fibrils. An unbiased search for the sequence determinants of amyloid- $\beta$ fibrillogenesis revealed that Phe-19 was particularly important for forming fibrils $(19,23)$. The structure of the fibrils formed from the central region of amyloid- $\beta$ (residues 11-25) shows intersheet stacking of phenylalanine rings (13). In the case of the type 2 diabetes-related amyloid peptide, islet amyloid polypeptide, the truncated peptides NFGAIL and FGAIL form fibrils similar to the full-length fibril (24), whereas GAIL does not form fibrils. Near-UV circular dichroism spectra recorded immediately before insoluble fibrils showed stacking of aromatic rings in islet amyloid polypeptide (25), and an alanine scan of the NFGAIL sequence revealed the importance of the phenylalanine residues in amyloid fibril formation (26). Molecular simulations of NFGAIL models show that the phenylalanine rings cement together the macromolecular assemblies (27). Aromatic character was found to be the likely factor determining whether short fragments of human calcitonin (28) (medullary thyroid carcinoma) formed amyloid. Solidstate NMR spectroscopy of a pentapeptide of human calcitonin (Table 1 , DFNKF) has revealed a possible $\pi-\pi$ stacking arrangement between phenylalanine between $\beta$-strands in a $\beta$-sheet (29). Similar experiments have also taken place with the precursor to aortic medial amyloid (30) and serum amyloid A (chronic inflammation amyloidosis) (31), which contains four phenylalanine residues in the first 15 residues, including one FF motif. Studies of short fragments of $\beta-2$ microglobulin revealed that aromatic residues capable of $\pi-\pi$ stacking were important for fibril formation (32). Short sequences containing aromatic residues are present in $\mathrm{PrP}$, a protein associated with Creutzfeldt-Jakob disease and in the yeast prion, Sup35p. Collectively, this evidence suggests that aromatic residues are likely to play an important role in the process of amyloidogenesis (9).

1. Eanes, E. D. \& Glenner, G. G. (1968) J. Histochem. Cytochem. 16, 673-677.

2. Geddes, A. J., Parker, K. D., Atkins, E. D. T. \& Beighton, E. (1968) J. Mol. Biol. 32, 343-358.

3. Astbury, W., Dickinson, S. \& Bailey, K. (1935) Biochem. J. 29, 2351-2360.

4. Dobson, C. (2001) Phil. Trans. Soc. London Ser. B 356, 133-145.

5. Hirota-Nakaoka, N., Hasegawa, K., Naiki, H. \& Goto, Y. (2003) J. Biochem. (Tokyo) 134, 159-164.

6. Sunde, M., Serpell, L., Bartlam, M., Fraser, P., Pepys, M. \& Blake, C. (1997) J. Mol. Biol. 273, 729-739.

7. Diaz-Avalos, R., Long, C., Fontano, E., Balbirnie, M., Grothe, R., Eisenberg, D. \& Caspar, D. L. D. (2003) J. Mol. Biol. 330, 1165-1175.

8. Hosia, W., Bark, N., Liepinish, E., Tjernberg, A., Persson, B., Hallen, D., Thyberg, J., Johansson, J. \& Tjernberg, L. (2004) Biochemistry 43, 4655-4661.

9. Gazit, E. (2002) FASEB J. 1, 77-83.

10. Tjernberg, L., Hosia, W., Bark, N., Thyberg, J. \& Johansson, J. (2002) J. Biol. Chem. 277, 43243-43246.

11. Serpell, L. C., Fraser, P. E. \& Sunde, M. (1999) in Methods in Enzymology, ed. Wetzel, R. (Academic, New York), Vol. 309, pp. 526-536.

12. Lovell, S. C., Davis, I. W., Arendall, W. B., III, De Bakker, P. I. W., Word, J. M., Prisant, M. G., Richardson, J. S. \& Richardson, D. C. (2003) Proteins 50, 437-450.

13. Sikorski, P., Atkins, E. D. T. \& Serpell, L. C. (2003) Structure (Cambridge, MA, U.S.) 11, 915-926.

14. Sunde, M. \& Blake, C. (1998) Q. Rev. Biophys. 31, 1-39.

15. Jimenez, J. L., Guijarro, J. I., Orlova, E., Zurdo, J., Dobson, C., Sunde, M. \& Saibil, H. (1999) EMBO J. 18, 815-821.

16. Sun, S. \& Bernstein, E. R. (1996) J. Phys. Chem. 100, 13348-13366.

17. McGaughey, G. B., Gagne, M. \& Rappe, A. K. (1998) J. Biol. Chem. 273, 15458-15463.

18. De la Paz, M. \& Serrano, L. (2004) Proc. Natl. Acad. Sci. USA 101, 87-92.

19. Esler, W. P., Stimson, E. R., Ghilardi, J. R., Lu, Y.-A., Felix, A. M., Vinters, H. V., Mantyn, P. W., Lee, J. P. \& Maggio, J. E. (1996) Biochemistry 35, 13914-13921.

20. Tjernberg, L. O., Näslund, J., Lindqvist, F., Johansson, J., Karlström, A. R., Thyberg, J., Terenius, L. \& Nordstedt, C. (1996) J. Biol. Chem. 271, 8545-8548.
Aromatic stacking is important in many areas of structural biology, including the packing of the hydrophobic cores of proteins giving stabilization of the tertiary structure, host-guest interactions, porphyrin aggregation in solution and the stabilization of DNA by base packing (33). Aromatic interactions have been proposed to contribute to the unique properties of thermophilic proteins (34) and aromatic clusters have been implicated in conferring additional thermostability to thermophiles when compared with their mesophilic counterparts. These attractive nonbonded interactions are important in general molecular recognition and self-assembly (17, 35-37). Recently, assembly of a diphenylalanine dipeptide (38) resulted in stable nanotubes that could then be coated in silver to produce nanowires. Presumably, the stacking of the aromatic side chains is important in the assembly and stability of these fibers.

Here, we show $\beta$-sheet ribbons stabilized by charge pairing and associated by means of a phenylalanine zipper. The staggered arrangement creates a highly stable crystalline structure with the capacity to extend in all three dimensions. The $\pi$-stacking provides an energetic contribution (39), which may drive the self-assembly process. The side-chain interactions result in specific directionality, order, and orientation, which leads to a highly ordered, stable, cross- $\beta$ structure rather than amorphous aggregate. Each amyloid-forming protein has a unique arrangement of side chains that will pack together in the core of the $\beta$-sheet structure. Many amyloidogenic proteins show a high propensity for aromatic and charged residues. The specific nature of the side-chain interactions for each protein or polypeptide will drive the rate of fibril formation as well as the resulting fiber stability. This structure highlights the importance of intersheet side-chain interactions for amyloid formation, order, and stability.

We thank Dr. G. Chavali and S. MacDonald for help with data collection and Dr. J. Elliot for help with data analysis. Synchrotron data were collected at the European Synchrotron Radiation Facility (Grenoble, France). L.C.S. is supported by a Wellcome Trust Research Career Development Fellowship. O.S.M. holds a Medical Research Council Studentship.

21. Findeis, M. A., Musso, G. M., Arico-Muendel, C. C., Benjamin, H. W., Hundal, A. M., Lee, J., Chin, J., Kelley, M., Wakefield, J., Hayward, N. \& Molineaux, S. M. (1999) Biochemistry 38, 6791-6800.

22. Soto, C., Sigurdsson, E. M., Morelli, L., Kumar, R. A., Castano, E. M. \& Frangione, B. (1998) Nat. Med. 4, 822-826.

23. Wurth, C., Guimard, N. K. \& Hecht, M. H. (2002) J. Mol. Biol. 319, 1279-1290.

24. Tenidis, K., Waldner, M., Bernhagen, J., Fischle, W., Bergmann, M., Weber, M., Merkle, M., Voelter, W., Brunner, H. \& Kapurniotu, A. (2000) J. Mol. Biol. 295, 1055-1071.

25. Kayed, R., Bernhagen, J., Greenfield, N., Sweimeh, K., Brunner, H., Voelter, W. \& Kapurniotu, A. (1999) J. Mol. Biol. 287, 781-796.

26. Azriel, R. \& Gazit, E. (2001) J. Biol. Chem. 276, 34156-34161.

27. Zanuy, D., Porat, Y., Gazit, E. \& Nussinov, R. (2004) Structure (Cambridge, MA, U.S.) 12, 439-455.

28. Reches, M., Porat, Y. \& Gazit, E. (2002) J. Biol. Chem. 277, 35475-35480.

29. Naito, A., Kamihira, M., Inoue, R. \& Saito, H. (2004) Magn. Reson. Chem. 42, 247-257.

30. Häggqvist, B., Näslund, J., Sletten, K., Westermark, G. T., Mucchiano, G., Tjernberg, L. O., Nordstedt, C., Engström, U. \& Westermark, P. (1999) Proc. Natl. Acad. Sci. USA 96, 8674-8669.

31. Westermark, G., Engström, U. \& Westermark, P. (1992) Biochem. Biophys. Res. Commun. 182, 27-33.

32. Jones, S., Manning, J., Kad, N. M. \& Radford, S. E. (2003) J. Mol. Biol. 325, 249-257.

33. Waters, M. L. (2002) Curr. Opin. Chem. Biol. 6, 736-741.

34. Kannan, N. \& Vishveshwara, S. (2000) Protein Eng. 13, 753-761.

35. Claessens, C. G. \& Stoddart, J. F. (1997) J. Phys. Org. Chem. 10, 254-272.

36. Shetty, A. S., Zhang, J. \& Moore, J. S. (1996) J. Am. Chem. Soc. 118, 1019 1027.

37. Gillard, R. E., Raymo, F. M. \& Stoddart, J. F. (1997) Chem. Eur. J. 3, 1933-1940.

38. Reches, M. \& Gazit, E. (2003) Science 300, 625-627.

39. Hunter, C. A. (1993) Angew. Chem. Int. Ed. 32, 1584-1586. 